\title{
Melatonin decreases androgen-sensitive prostate cancer growth by suppressing SENP1 expression
}

\author{
Lin Hao ${ }^{1,2 \#}$, Yang Dong ${ }^{1,2 \#}$, Jun-Jie Zhang ${ }^{1,2 \#}$, Hou-Guang $\mathrm{He}^{2}$, Jian-Gang Chen ${ }^{1}$, Shao-Qi Zhang ${ }^{1}$, \\ Qian-Jin Zhang ${ }^{1}$, Wei Wu ${ }^{1}$, Cong-Hui Han ${ }^{1,2 *}$, Zhen-Duo Shi ${ }^{2 *}$ \\ ${ }^{1}$ Medical College of Soochow University, Suzhou, China; ${ }^{2}$ Department of Urology, Xuzhou central hospital, Xuzhou, China \\ Contributions: (I) Conception and design: CH Han; (II) Administrative support: CH Han, ZD Shi; (III) Provision of study materials or patients: L \\ Hao, Y Dong, JJ Zhang; (IV) Collection and assembly of data: Y Dong, HG He, JG Chen; (V) Data analysis and interpretation: L Hao, QJ Zhang, \\ W Wu; (VI) Manuscript writing: All authors; (VII) Final approval of manuscript: All authors. \\ \#These authors contributed equally to this work and should be considered as co-first authors. \\ *These authors contributed equally to this work and should be considered as co-Corresponding Author. \\ Correspondence to: Cong-Hui Han. Medical College of Soochow University, Suzhou 215006, China; Department of Urology, Xuzhou central hospital, \\ Xuzhou 221009, China. Email: hanchdoctor@126.com; Zhen-Duo Shi. Department of Urology, Xuzhou central hospital, Xuzhou 221009, China. \\ Email: bujiniao2008@qq.com.
}

Background: Melatonin is a hormone naturally produced by the pineal gland in the brain. In addition to modulating circadian rhythms, it has pleiotropic biological effects including antioxidant, immunomodulatory, and anti-cancer effects. Herein, we report that melatonin has the ability to decrease the growth and metastasis of androgen-dependent prostate cancer.

Methods: To evaluate the anti-cancer effect of melatonin on androgen-sensitive prostate cancer in vitro or in vivo, the effects of cell proliferation, apoptosis, migration and invasion were analyzed by using 3-(4,5-dimethylthiazol-2-yl)-2,5-diphenyltetrazolium bromide (MTT), colony formation, flow cytometry, Transwell assay, and immunohistochemistry (IHC), respectively. Next, the interaction between androgen receptor (AR) and SUMO specific protease 1 (SENP1) was detected by real-time quantitative reverse transcription-polymerase chain reaction (qRT-PCR) and western blotting, and confirmed by luciferase reporter assay. Furthermore, the Small Ubiquitin-like Modifier (SUMO) proteins are a group of small proteins that are covalently attached to and detached from other proteins in cells to modify their function. (SUMOylation) of histone deacetylases 1 (HDAC1) was measured by proximity ligation assay (PLA).

Results: The treatment of melatonin cripples the transcriptional activity of AR, which is essential for the growth of the androgen-dependent prostate cancer cell, LNCaP. The lower activity of AR was dependent on melatonin induced SUMOylation of HDAC1, which has been established as a key factor for the transcriptional activity of AR. Mechanistically, the effect of melatonin on AR was due to the decreased SENP1 protein level and the subsequent increased HDAC1 SUMOylation level. The overexpression of SENP1 abrogated the anti-cancer ability of melatonin on LNCaP cells.

Conclusions: These findings indicate that melatonin is a suppressor of androgen-dependent prostate cancer tumorigenesis.

Keywords: Melatonin (MT); prostate cancer; cell growth; SUMO specific protease 1 (SENP1)

Submitted Nov 25, 2021. Accepted for publication Jan 14, 2022.

doi: $10.21037 /$ tau-21-1110

View this article at: https://dx.doi.org/10.21037/tau-21-1110 


\section{Introduction}

Melatonin (MT), chemically N-acetyl-5-methoxy tryptamine, is a major player in the coordination of circadian rhythms and seasonal breeding in photoperiodic species (1-3). In addition to adjusting the timing of the body's circadian clock, MT acts as a neuromodulator, cytokine, biological response modifier, and antioxidant (4-7). Furthermore, research on MT has revealed a potential anti-cancer activity of MT in a variety of cancers, including prostate, breast, lung, liver, colon, etc. (8-14). The oncostatic actions of MT include induction of apoptosis, anti-proliferation, suppression of invasion and metastasis, anti-angiogenesis, and direct augmentation of immunomodulation (15). For example, in the literature, acting through its receptors MT1, MT inhibits PLC/p38c-Jun signaling pathway to repress expression of MMP13 and prostate carcinoma cells proliferation, migration and invasiveness (16). The cellular action of MT is achieved via a receptor-dependent or receptor-independent manner $(17,18)$. At the cell surface, two well-characterized G-protein-coupled 7 -pass membrane receptors are responsible for the signal transduction of $\mathrm{MT}(19,20)$. In addition to the membrane receptor-dependent pathway, MT has shown affinity to the orphan nuclear receptor $\mathrm{RZR} / \mathrm{ROR} \alpha$, which is prominently expressed both in the periphery and the brain (21). Based on the aforementioned literature, the mechanisms of melatonin action on prostate cancer attracted our research interest and attention.

Prostate cancer is the most common non-cutaneous cancer among American men, with more than 180,890 new cases in the United States in 2016 (22). It is characterized by the most rapid increase in incidence of all malignancies in older men. Research on prostate cancer has shown that overactive androgen promotes early prostate cancer tumorigenesis in an androgen receptor (AR)-dependent manner (23). Consequently, androgen deprivation therapy involving surgical or chemical castration has proven to be the standard first-line option for patients with prostate cancer (24). However, the disease will generally recur and become androgen-independent for growth within 1-2 years of androgen deprivation therapy. The progression of prostate cancer was also found to rely on AR signaling sustained by adrenal and intratumoral androgens, as well as the upregulation of $\mathrm{AR}$ protein expression in tumor cells (25). Furthermore, MT could be involved in regulation of AR. The literature suggest that MT reduces AR production in granulosa cells from polycystic ovary syndrome patients (26), and MT inhibits expression of AR and promotes the translocation of $\mathrm{AR}$ into the nucleus in prostate cancer cells (27). In general, AR signaling is the key regulator for the initiation and progression of prostate cancer.

Recently, a case-cohort study of 928 Icelandic men showed that lower levels of urinary 6-sulfatoxymelatonin were associated with an increased risk of advanced prostate cancer (28). Serum MT levels were also lower in prostate cancer patients than in age-matched controls (29). Membrane MT receptors have been found in benign prostate epithelial cells, which provides evidence for the involvement of MT in control cell growth and viability (30), and indeed, MT exerts an anti-tumor effect on prostate cancer both in vitro and in vivo (31). MT has demonstrated its strong effect on limiting androgen-sensitive prostate cancer LNCaP cell growth (32). However, the molecular mechanism of this regulation remains unclear.

Accumulated evidence has shown that the abnormal proliferation of LNCaP cells was linked to a significantly elevated expression of the human sentrin/SUMO-specific protease 1 (SENP1), which modulated the transcriptional activity of ARs by manipulating the SUMOylation level of histone deacetylases 1 (HDAC1). SENP1 can remove SUMO from modified HDAC1 and reduces its deacetylase activity, thereby decreasing HDAC1's repressive effect on AR-dependent transcription (33-35). SUMO modification is a dynamic process that regulates the target protein's activation, function, and/or subcellular localization. In the dynamic cycle of SUMOylation $(36,37)$, SENPs are the key factors in the regulation of the physiological and pathological process mediated by SUMOylated proteins $(38,39)$. Moreover, SENP1 contributes to the development and progression of prostate cancer. Modulating SENP1 level dictates colony formation of prostate cancer cell lines, tumor growth in nude mice and also prostate cancer cell migration and invasion (40). SENP1 interacts with and deSUMOylates CSR1 to prevent its degradation and enhances CSR1-dependent prostate cancer cell death (41). Herein, we present evidence that MT inhibits the proliferation of $\mathrm{LNCaP}$ cells by decreasing the expression of SENP1, which is required for the full activation of ARs. In light of the negative control of androgen signaling, MT may be an effective treatment for androgen-dependent prostate cancer. We present the following article in accordance with the ARRIVE reporting checklist (available at https://tau.amegroups.com/article/view/10.21037/tau$21-1110 / \mathrm{rc})$. 


\section{Methods}

\section{Chemicals and cell cultures}

MT, R1881 (synthetic androgen), luzindole, and all the primary antibodies were purchased from Sigma-Aldrich (St Louis, MO, USA). Secondary antibodies were purchased from Santa Cruz Biotechnology (Santa Cruz, CA, USA). Roswell Park Memorial Institute (RPMI) 1640 medium and fetal bovine serum (FBS) were obtained from Gibco (Invitrogen Life Technologies, Grand Island, NY, USA).

The human prostate cancer cell line, LNCaP, was obtained from American Type Cell Culture (ATCC, Rockville, MD, USA). The growth medium used for LNCaP was RPMI 1640 containing $10 \%$ FBS with 100 units/mL penicillin-streptomycin.

\section{MTT assay}

For the cell proliferation assay, 5,000 cells/well were seeded in triplicate in 96-well plate. The plate was incubated for 2 days in $37^{\circ} \mathrm{C}$ and $5 \%$ carbon dioxide $\left(\mathrm{CO}_{2}\right)$ humidified chamber. $10 \mu \mathrm{L}$ 3-(4,5-dimethylthiazol-2-yl)-2,5-diphenyltetrazolium bromide (MTT, $10 \mathrm{mg} / \mathrm{mL}$ ) were added and incubated in $37^{\circ} \mathrm{C}$ for $2 \mathrm{~h}$. The MTT was then discarded and added $100 \mu \mathrm{L}$ Dimethyl Sulphoxide (DMSO, Sigma, Germany) to each well. The absorbance at $570 \mathrm{~nm}$ was measured using microplate reader (Thermo Fisher, US).

\section{Apoptosis assay}

After collection, the cells were resuspended with $200 \mu \mathrm{L}$ $1 \times$ binding buffer. Then, $5 \mu \mathrm{L}$ Annexin $\mathrm{V}$-fluorescein isothiocyanate (FITC) was added and incubated for $15 \mathrm{~min}$ at room temperature in the dark. Next, the cells were gently vortexed and $5 \mu \mathrm{L}$ Prodium Iodide (PI) was added. The cells were incubated for $5 \mathrm{~min}$ protecting from light. Then, $300 \mu \mathrm{L} 1 \times$ binding buffer was added and flow cytometry was performed within $1 \mathrm{~h}$.

\section{Migration and invasion assay}

The most optimal MT concentration (2 $\mathrm{mM}$ ) was determined by MTT and apoptosis experiments, we used $2 \mathrm{mM}$ concentration for subsequent experiments in vitro. The cell migration and invasion were detected using the Transwell plates and cell inserts (BD Biosciences, San Jose, CA). For cell migration assay, the LNCaP cells treated with MT or MT and luzindole were appropriately $\left(5 \times 10^{5}\right.$ cell/well $)$ seeded into the cell insert with serum-free media and $10 \%$ FBS was used as a chemoattractant. For cell invasion assay, each cell insert was needed precoated with $100 \mu \mathrm{L}$ Matrigel (Corning, Tewksbury, MA, USA). The other steps were similar to cell migration assay. After $22 \mathrm{~h}$ incubation at $37^{\circ} \mathrm{C}$, the cells on the bottom side of the cell insert were fixed, stained with Diff-Quik staining kit (Sysmex, Japan). Briefly, the cells were fixed Diff-Quik Fixative, Diff-Quik Solution I, and then Diff-Quik Solution II were used in this order at their original concentrations for $10 \mathrm{~min}$ at room temperature. Subsequently, the pictures of each group were taken under $\times 200$ magnification using a microscope. (Zeiss, Germany), and the cell number was counted in three random fields of view.

\section{Scratch wound bealing assay}

In brief, the cells were seeded and grown to confluence on 6-well plate. A scratch wound of was created in the confluent cell layer by using a P200 pipette tip. The floating cells were removed by washing three time with PBS. The control group was treated with medium alone and the test groups were treated with the corresponding agents. Images (×100 magnification) were photographed at $24 \mathrm{~h}$ and measured using ImageJ software.

\section{Soft-agar assay}

In total, 500 cells were plated in complete medium in sixwell plates, with a bottom layer of $0.6 \%$ agarose and a top layer of $0.3 \%$ agarose. The medium was replaced every 3 days. After 28 days, the cells were fixed with $95 \%$ methanol and stained with Giemsa (Sigma).

\section{Quantitative real-time polymerase chain reaction (PCR) assay}

Total Ribonucleic Acid (RNA) of cells was extracted using the TRIzol reagent (Invitrogen) following the manufacturers instruction and quantified by absorbance at $260 \mathrm{~nm}$. Next, the mRNA was converted to Firststrand complementary DNA (cDNA) using the RevertAid ${ }^{\mathrm{TM}}$ Reverse Transcriptase kit (Thermo Scientific TM Life Technologies, NY, USA). The primer sequences used for PCR were as follows: SENP1 forward 5'-CTCCAACTCCCAGTTCTACTTTC-3', reverse 5'-GTTCTTCAATCTGGCGCAATC-3'; AR forward 5'-GGTTACACCAAAGGGCTAGAA-3', reverse 5'-GACTTGTAGAGAGAC AGGGTAGA-3'; 
Prostate-Specific Antigen (PSA) forward 5 ' - CCCACTGCATCAGGAACAA-3', reverse 5'-GCTGTGGCTGACCTGAAATA-3'; $\beta$-actin forward 5 '-CATGTACGTTGCTATCCAGGC-3', reverse 5'-CTCCTTA ATGTCACGCACGAT-3'.

\section{Western blot analysis}

Protein expression was assessed by Western blot. In brief, the cells were lysed with Radio-Immunoprecipitation Assay (RIPA) buffer and the total cell protein concentrations were measured by the Bradford method (Bio-Rad, Hercules, CA, USA).

Total cell extracts were obtained by lysing the cells in Radio-Immunoprecipitation Assay (RIPA) buffer. Protein concentration was measured using the Bradford assay (BioRad, Hercules, CA, USA). Cellular proteins were extracted and separated in $4-10 \%$ Tris glycine/sodium dodecyl sulphate (SDS)-polyacrylamide gels and transferred to nitrocellulose membranes. The membranes were blocked with $5 \%$ non-fat milk and incubated with corresponding primary and secondary antibodies. Finally, the blots were visualized with chemiluminescence ECL reagent and the $\beta$-actin protein was used as the endogenous control.

\section{Animal model}

Male BALB/c nude mice, 5 weeks of age, were housed. To evaluate the therapeutic efficacy of MT in a human LNCaP xenograft mouse model, LNCaP cells $\left(10^{8}\right.$ in $100 \mu \mathrm{L}$ PBS $)$ were injected subcutaneously into two bilateral sites on the lower back of the nude mice.

The mice were randomly assigned to either the control (vehicle treated, $\mathrm{n}=5$ ) or MT administration ( $\mathrm{n}=5$ ) groups. The vehicle solution was prepared with $9 \mathrm{~mL}$ of PBS and $1 \mathrm{~mL}$ of DMSO. The MT administration group mice received an intraperitoneal injection (IP) of $100 \mu \mathrm{L}$ of MT solution ( $40 \mathrm{mg} / \mathrm{kg}$ of body weight) for 5 days a week, while the control group mice received the same volume of vehicle solution intraperitoneally. MT treatment was given $1 \mathrm{~h}$ before the room lighting was switched off. MT administration prior to the nocturnal increase in endogenous MT may be most effective because tissues are most sensitive to the hormone at this time $(42,43)$.

The MT or vehicle were administered immediately after tumor cells implantation and continued for 35 days. On the $36^{\text {th }}$ day, the tumors were collected for immunohistochemistry (IHC) after euthanasia. This study was approved by the Ethics Committee of the Xuzhou Central Hospital (No. XZXY-LJ-20210303-024). The operation process of all experimental animals was strictly in accordance with the "Guide to the Care and Use of Experimental Animals” (2016) (44).

\section{Proximity ligation assay (PLA)}

In general, $24 \mathrm{~h}$ after transfection with hemagglutinin (HA)-HDAC1, Flag-SUMO1, or regulator of G protein signaling (RGS)-SENP, the $293 \mathrm{~T}$ cells were treated with $2 \mathrm{mM}$ MT for another $24 \mathrm{~h}$. Then, the harvested cells were fixed in $4 \%$ formaldehyde and permeabilized with $0.3 \%$ Nonidet P (NP)-40. PLA was performed using Duolink ${ }^{\circledR}$ InSitu Detection Reagents Red (Sigma). The pictures were captured with confocal microscopy LSM710 (Zeiss, Germany).

\section{Statistical analysis}

Data were presented as mean \pm standard deviation. Statistical analysis was performed using the Student's $t$-test between two groups and one-way analysis of variance (ANOVA) NewmanKeuls post hoc tests for comparisons between more than two groups. A significance level of $\mathrm{P}<0.05$ was used.

\section{Results}

\section{MT suppresses LNCaP cell proliferation in vitro and in vivo}

To study the anti-cancer effect of MT on androgensensitive prostate cancer, we first employed the MTT assay to test the proliferation of $\mathrm{LNCaP}$ cells with MT treatment. After $48 \mathrm{~h}$ of MT treatment $(0.01-3.0 \mathrm{mM})$, the proliferation of $\mathrm{LNCaP}$ cells was significantly inhibited in a dose-dependent manner (Figure 1A). To investigate whether the decreased cell viability is associated with increased apoptosis, LNCaP cells were analyzed by Annexin V/PI staining-based flow cytometry at $48 \mathrm{~h}$ after MT treatment. As shown in Figure $1 B, 1 C$, the decreased proliferation of LNCaP cells was partially due to an increase in apoptosis induced by MT. Since LNCaP cells were androgen-sensitive cells, we questioned whether MT has effect on androgeninduced hyperproliferation of LNCaP cells. Seven days treatment with $10 \mathrm{nM} \mathrm{R} 1881$ (synthetic androgen) induced a 15 -fold increase in $\mathrm{LNCaP}$ cell proliferation. However, the proliferating effect of androgen on $\mathrm{LNCaP}$ cells was significantly restrained by MT (Figure 1D). 

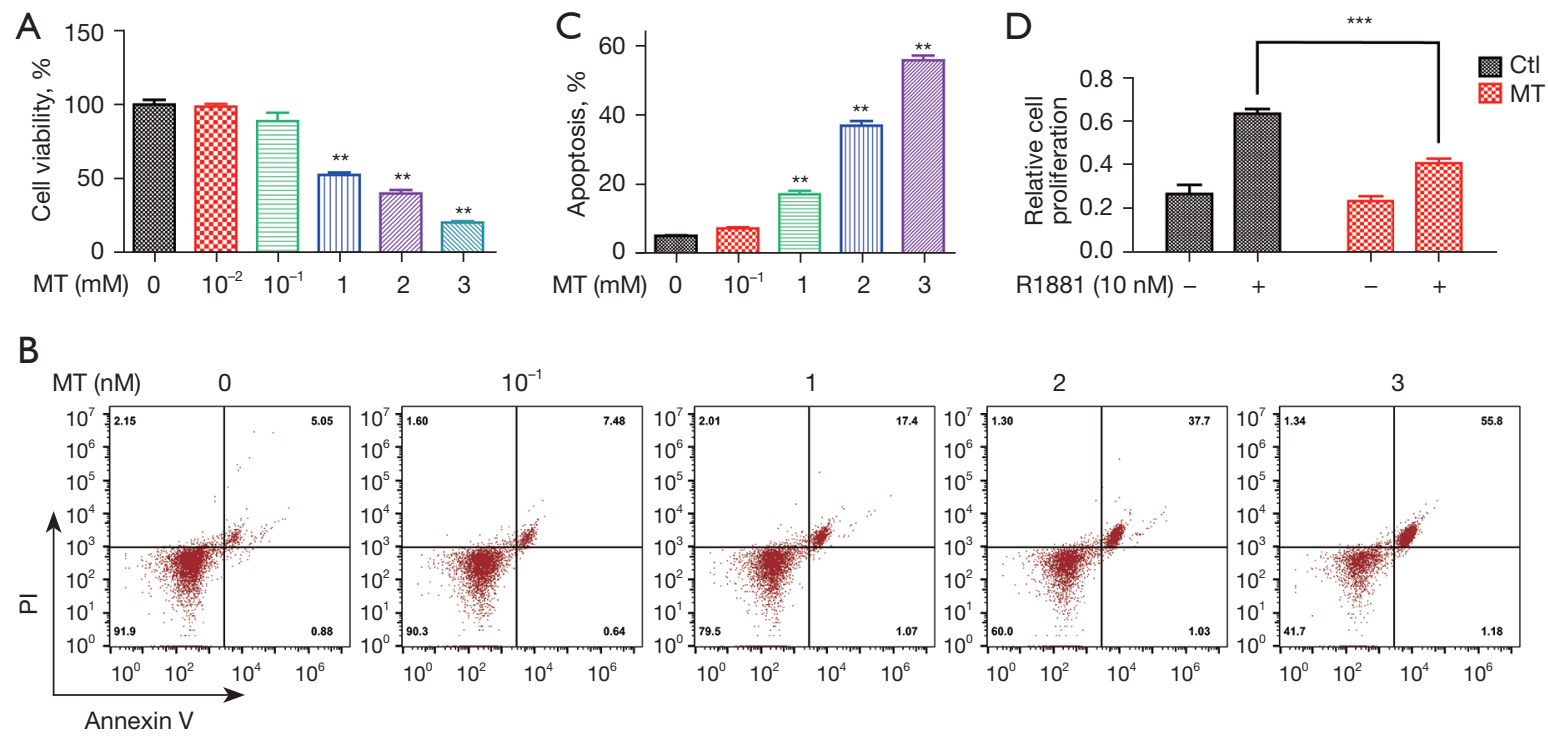

Figure $1 \mathrm{MT}$ suppresses LNCaP cell proliferation. (A) MTT assay for LNCaP cells treated with MT at the indicated doses for 48 h; DMSO was used as control; (B) flow cytometry analysis and (C) quantification of apoptosis induced by MT in LNCaP cells. (D) Cell number quantification showing the effect of $2 \mathrm{mM} \mathrm{MT}$ on androgen-mediated LNCaP cell proliferation. Data are presented as the mean \pm SEM. of three separated experiments. ${ }^{* *} \mathrm{P}<0.01,{ }^{* * *} \mathrm{P}<0.001$ versus control group. MT, melatonin; LNCaP, Lymph Node Carcinoma of the Prostate; MTT, 3-(4,5-dimethylthiazol-2-yl)-2,5-diphenyltetrazolium bromide; DMSO, dimethyl sulfoxide.

To further demonstrate the anti-cancer effect of MT on androgen-sensitive prostate cancer, we queried whether MT treatment inhibits the ability of LNCaP cells to form anchorage-independent foci in culture or xenograft tumors in nude mice. After plating approximately $500 \mathrm{LNCaP}$ cells in a $60 \mathrm{~mm}$ petri dish and culturing for 28 days, we observed approximately 55 colonies of LNCaP cells without MT treatment, while the cells pre-treated with $2 \mathrm{mM} \mathrm{MT}$ for $48 \mathrm{~h}$ generated no more than 30 colonies (Figure $2 A, 2 B$ ). The in vivo effect of $M T$ was investigated by performing a xenograft tumor experiment; approximately $10^{8} \mathrm{LNCaP}$ cells were injected subcutaneously into two bilateral sites on the lower back of 5-week-old male BALB/c nude mice who were then treated with MT $(40 \mathrm{mg} / \mathrm{kg}$ ) or vehicle for 35 days. After monitoring the tumor for 5 consecutive weeks, MT-treated mice showed significantly smaller tumors in contrast to vehicle-treated mice (Figure 2C,2D). On histological sections, xenograft tumors with MT treatment showed decreased staining for Ki67, suggesting a relatively low growth (Figure 2E).

\section{MT suppresses LNCaP cell migration and invasion}

The LNCaP cell line was established from a metastatic lesion of human prostatic adenocarcinoma. To determine whether MT has an effect on metastasis, we examined the migration and invasion ability of LNCaP cells with MT or MT and luzindole (the MT receptor antagonist) treatment. As shown in Figure 3A,3B, MT inhibited the invasion of LNCaP cells via a Matrigel-coated filter by two- to threefold, while additional treatment with luzindole significantly abolished the effect of MT.

The migration of LNCaP cells was measured using the wound-healing assay. At $24 \mathrm{~h}$ after being scrubbed, cells with MT treatment showed a decreased migration ability; however, this situation could be markedly reversed by luzindole (Figure 3C). Further investigation using the Boyden chamber assay confirmed that MT strongly alleviated the migration of LNCaP cells (Figure 3D,3E).

\section{MT suppresses the transcriptional activity of $A R$}

Since AR activity is essential for the proliferation, migration, and invasion of $\mathrm{LNCaP}$ cells, we investigated whether MT could decrease AR activity. We preformed a luciferase assay to evaluate the repression effect of MT. When co-transfected with $\mathrm{AR}$, the antioxidant response element (ARE)-luciferase reporter showed a robust inductive response to $A R$, but $M T$ treatment for $48 \mathrm{~h}$ significantly repressed this induction in a concentration-dependent manner (Figure $4 A$ ). To further 
A

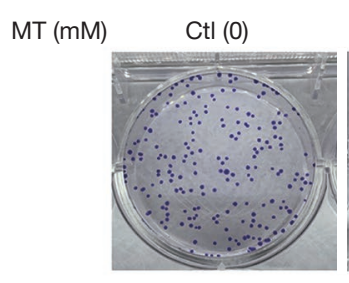

D

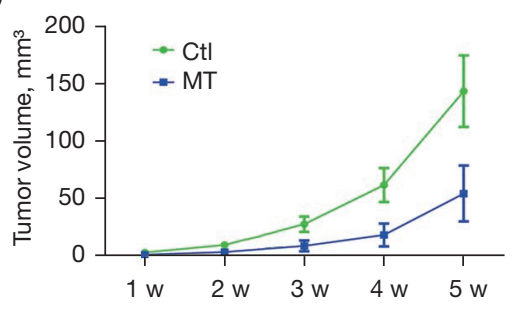

B

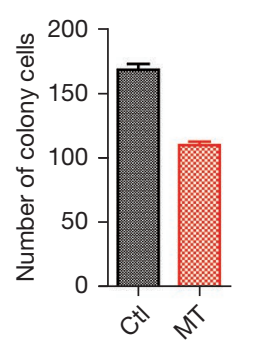

E

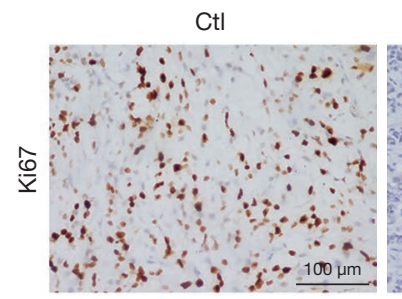

C

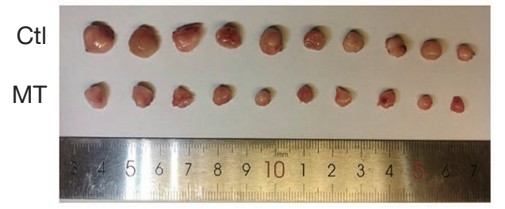

MT

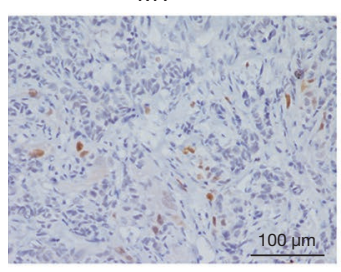

Figure 2 Effects of MT on the tumorigenesis of prostate cancer cells. (A) Soft-agar assay showing reduced tumorigenesis of LNCaP cells treated with MT. (B) Statistical analysis of the colony number from each group in (A). (C) Anti-tumor activity of MT in vivo. Fiveweek-old male BALB/c nude mice were injected subcutaneously with a phosphate buffer solution suspension containing 108 log-phase LNCaP cells and then treated with MT $(40 \mathrm{mg} / \mathrm{kg})$ or vehicle for 5 weeks. Each test and corresponding control lines were injected into five individual mice. (D) The mice were monitored weekly and the tumor volume was measured as an index of the growth rate. Tumor volume was calculated using the equation volume $=1 / 2\left(\right.$ length $\times$ width $\left.^{2}\right)$. The data are presented as mean \pm SEM. (E) IHC staining with Ki-67 in vehicle- and MT-treated tumors. MT, melatonin; LNCaP, Lymph Node Carcinoma of the Prostate.

ascertain the inhibitive ability of MT, we analyzed the induction of PSA, a well-known AR target, in $\mathrm{LNCaP}$ cells with or without MT treatment. The induction of PSA was reduced with MT treatment, compared to that in the control cells (Figure 4B). However, there was no difference in AR expression (Figure 4C). Likewise, western blot analyses of AR and PSA expression produced results that were essentially consistent with the Messenger RNA (mRNA) measurements (Figure 4D).

\section{MT modulates AR activity by decreasing SENP1 expression}

SENP1 has been shown to play a prominent role in the regulation of AR-dependent transcription and $\mathrm{LNCaP}$ cell proliferation (30). Given that SENP1 can regulate the activity of AR, we reasoned that SENP1 might involve in the inhibitory effect of MT on LNCaP cells. We first examined the effect of MT on the expression of SENP1 with RT-qPCR, and found that SENP1 was surely repressed by MT treatment (Figure 5A). Western blot analysis of SENP1 in LNCaP cells treated with MT coincided with the mRNA level (Figure 5B). Similar to our previous results, mRNA and protein expression were both decreased during MT treatment in a dose-dependent manner (Figure 5A,5B).
To further investigate the effect of MT on SENP1, we analyzed the mRNA and protein level in LNCaP cells with $2 \mathrm{mM}$ MT treatment at different time points. The results (as shown in Figure 5C, 5D) indicated that MT exerted a timedependent inhibitory effect on SENP1, while luzindole could eliminate the effect of MT. More importantly, the expression in xenograft tumor with MT treatment showed decreased staining for SENP1 (Figure 5E).

To investigate whether SENP1 has a role in MTmediated decrease of AR activity, we forced exogenous SENP1 expression in LNCaP cells, followed by $2 \mathrm{mM} \mathrm{MT}$ treatment for additional $24 \mathrm{~h}$, the mRNA and protein level of PSA was partially recovered (Figure 5F,5G). Consistent with our speculation, overexpression of SENP1 weakened the effect of MT on AR transcriptional activity (Figure 5H). The catalytic activity of SENP1 is required for this effect, as the catalytically inactive mutant SENP1 cannot antagonize MT (Figure 5H).

\section{MT induces SUMOylation of HDAC1}

As a SUMO specific protease, the primary function of SENP1 is to remove SUMO proteins from the substrate. It was reported that SENP1 could reverse the SUMOylation 
A

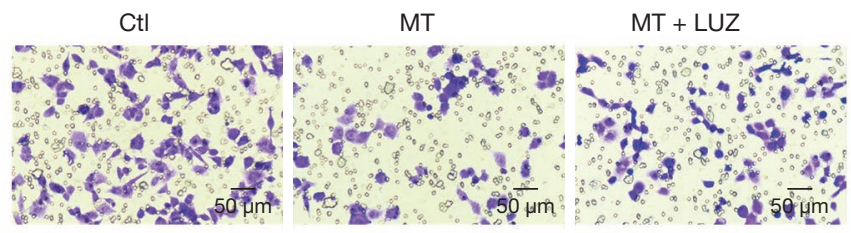

C
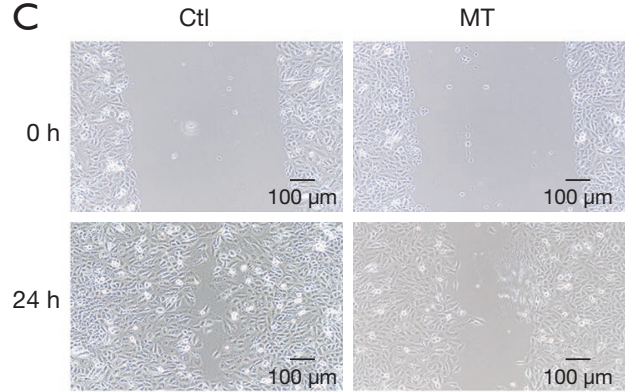

$M T+L U Z$

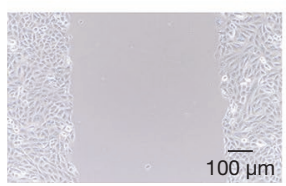

$\mathrm{D}$

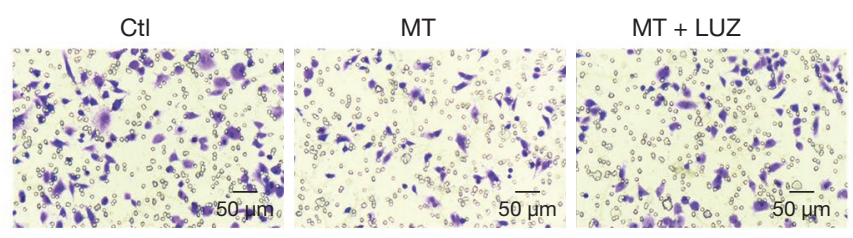

B

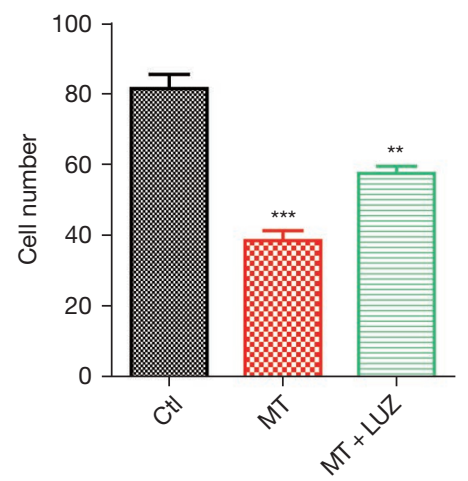

$\mathrm{E}$

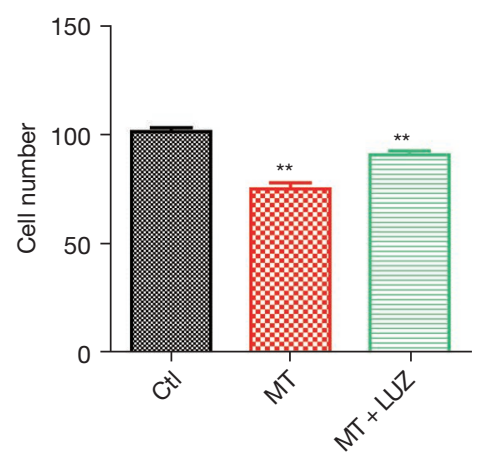

Figure $3 \mathrm{MT}$ inhibits LNCaP cell migration and invasion. (A) The effects of MT treatment ( $2 \mathrm{mM})$ or the combination of $\mathrm{MT}$ and the MT receptor antagonist, luzindole $(1 \mu \mathrm{M})$, for $48 \mathrm{~h}$ on LNCaP cell invasion was analyzed using a Matrigel Transwell invasion assay system (The cells stained with Diff-Quik staining kit. Briefly, the cells were fixed Diff-Quik Fixative, Diff-Quik Solution I, and then Diff-Quik Solution II were used in this order at their original concentrations for $10 \mathrm{~min}$ at room temperature. The pictures of each group were taken under $\times 200$ magnification using a microscope). (B) Statistical analysis of the invasion number from each group in (A). (C) Scratch wound healing assay of MT on LNCaP cells. (D) The effects of MT treatment $(2 \mathrm{mM})$ or the combination of MT and the MT receptor antagonist, luzindole $(1 \mu \mathrm{M})$, for $48 \mathrm{~h}$ on LNCaP cell migration was analyzed in a Transwell chamber (8-mm pore size membrane, BD Biosciences). (E) Statistical analysis of the migration number from each group in (D). The data in (B) and (E) are presented as the means \pm SEM. of three independent experiments. ${ }^{* *} \mathrm{P}<0.01,{ }^{* * *} \mathrm{P}<0.001$ versus control group. MT, melatonin; LNCaP, Lymph Node Carcinoma of the Prostate.

of HDAC1, a co-regulator in AR-dependent transcription system, and help to fine-tune the cellular responses to androgen. Combined with our previous results, we speculated that MT might block AR activity through SENP1dependent deSUMOylation. To support this notion, a PLA was conducted to determine the HDAC1 SUMOylation level after MT treatment. After co-transfection with HDAC1 and SUMO-1-expressing plasmids in 293T cells followed by MT treatment for $24 \mathrm{~h}$, we detected HDAC1 and SUMO-1 using two species-specific DNA-linked secondary antibodies. When HDAC1 and SUMO-1 were within 40nm of close proximity, ligation and polymerase amplification of the two DNA strands allowed for highly sensitive detection of SUMO-modified HDAC1. Using this assay, we detected more fluorescence signal than that of the vehicle-treated group (Figure 6A,6B). Further overexpression of SENP1 antagonized the SUMOylation induced action of MT (Figure 6C,6D).

In summary, our data indicated that MT engagement to AR-dependent signaling might have a strong effect on androgen-sensitive prostate cancer to retard cell proliferation, invasion, and metastasis. This regulation relies on SENP1, which modulates the SUMOylation level of HDAC1.

\section{Discussion}

Prostate cancer is the most frequently diagnosed malignancy and the leading cause of cancer mortality in men $(33,45)$. 


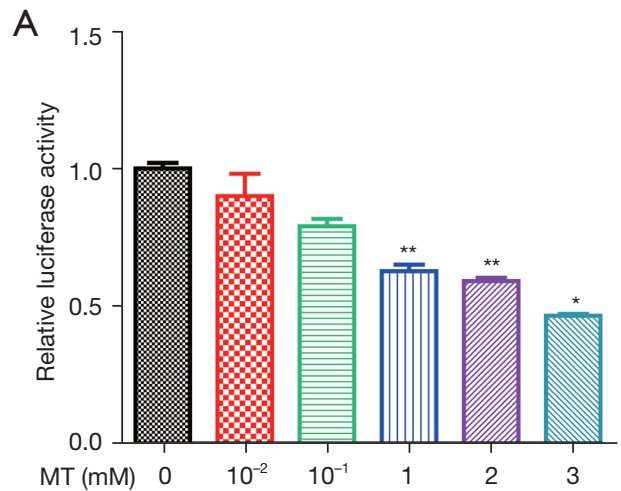

B
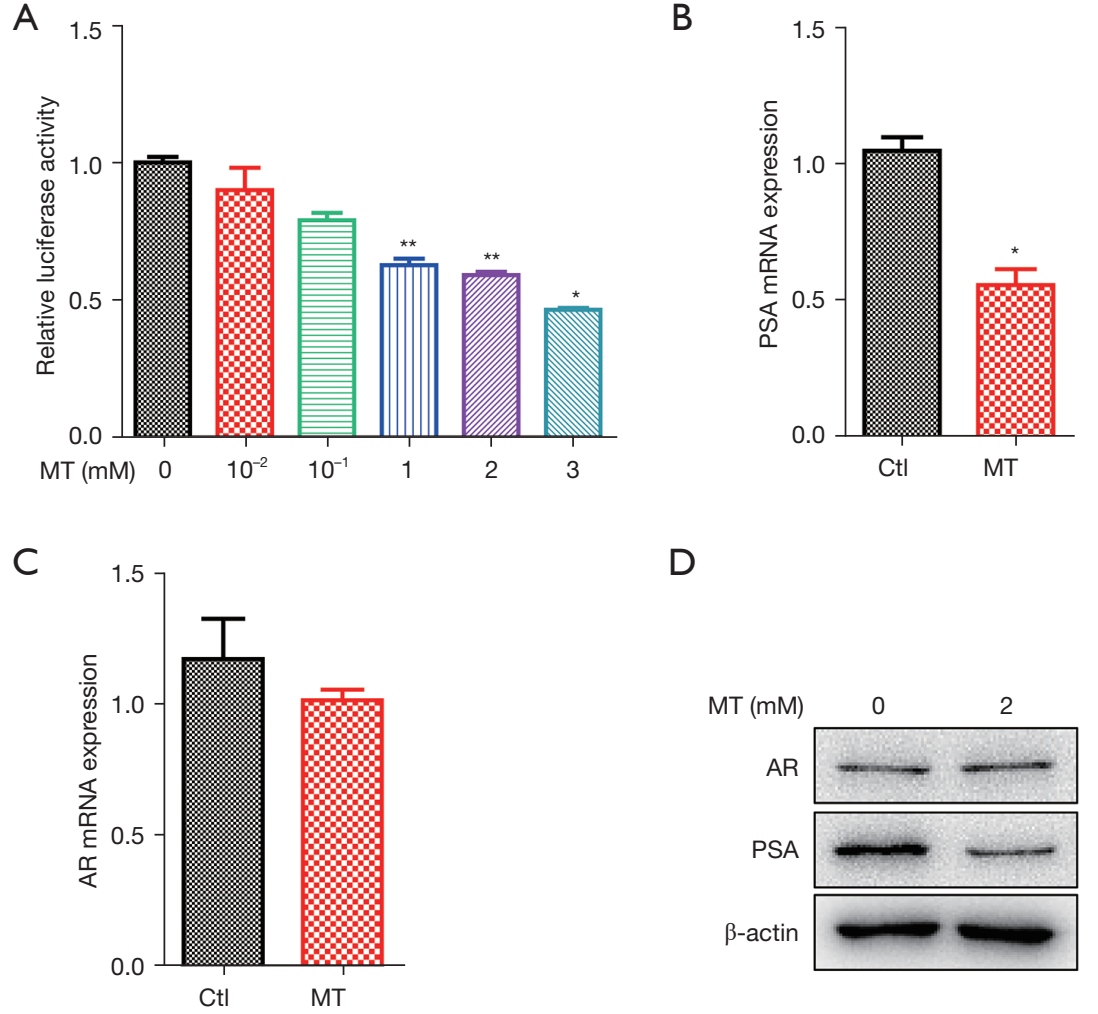

D

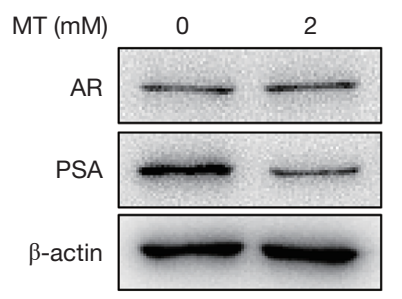

Figure 4 MT suppresses the transcriptional activity of AR. (A) 293T cells were co-transfected with AR expressing vector, ARE-luciferase reporter construct, and pRL-TK (internal control) for $24 \mathrm{~h}$, and then treated with MT at the indicated doses for an additional $48 \mathrm{~h}$. The transfected cells were harvested and assayed for luciferase activity. (B) RT-qPCR analysis of AR and (C) PSA transcript level in LNCaP cells treated with MT (2 mM) for $48 \mathrm{~h}$. (D) Western blot analysis of AR and PSA protein expression in LNCaP cells treated with MT (2 mM) for $48 \mathrm{~h}$. Data in (A-C) are presented as means \pm SEM. of three independent experiments. ${ }^{*} \mathrm{P}<0.05,{ }^{*} \mathrm{P}<0.01$ versus control group. MT, melatonin; AR, androgen receptor; ARE, AR-response elements; PSA, prostate-specific antigen; LNCaP, Lymph Node Carcinoma of the Prostate.

At the initial stages, prostate cancer is dependent upon androgens for growth, and is thus effectively combated by androgen deprivation therapy (ADT) (46). However, prostate cancer inevitably progresses to castration-resistant prostate cancer (CRPC) after a median duration of 1-2 years of ADT (47). This emphasizes the requirement for new therapeutic approaches. In this study, we investigated the effect of MT as well as the underlying mechanisms on LNCaP cells, which is a well-known androgen-sensitive prostate cancer cell line. MT strongly suppressed the cell proliferation, invasion, and metastasis of LNCaP cells both in vitro and in vivo. The effect of MT on $\mathrm{LNCaP}$ cells is mainly dependent on the regulation of AR transcriptional activity. Importantly, we demonstrated that MT could target SENP1 in tumor cells, which selectively modulates SUMO modification of HDAC1, followed by diminished transcriptional activity.
It is reported the key pathway involved in the progression from androgen-dependent prostate cancer to Castration Resistant Prostate Cancer (CRPC) under ADT may be the re-activation of the androgen- $\mathrm{AR}$ axis; for instance, that caused by AR mutation/overexpression/splice variants, altered expression of AR cofactors, and increased production of androgens (48). Furthermore, studies using human prostate cancer tissues have revealed a distinct transcriptional program in CRPC, and identified thousands of AR binding sites, most of which were not identified in prostate cancer cell lines (49). These publications highlight the importance of persistent AR signaling in prostate cancer, not only in the initiation but also in the progression. Our results showed that MT acted on HDAC1, blocked AR-dependent signaling transduction. Based on previous research and our results, we surmised that although we only inspected the function of MT on androgen-sensitive 

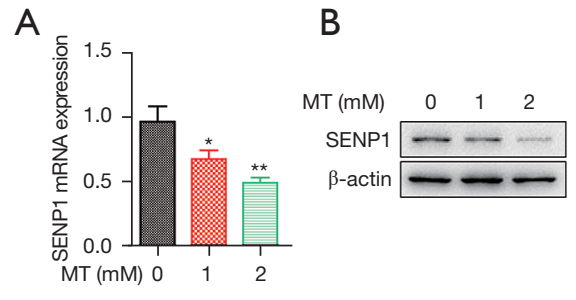

$\mathrm{F}$
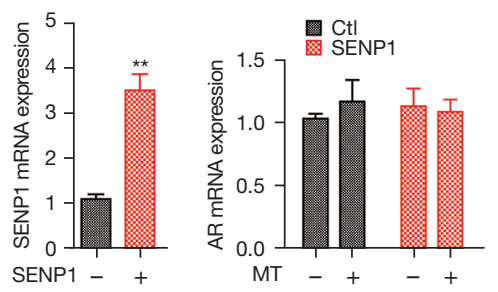

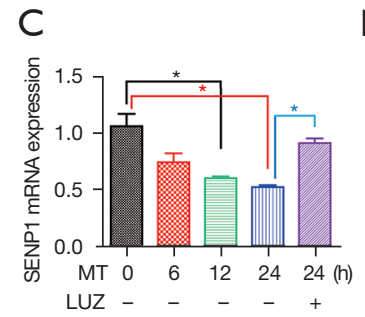

D

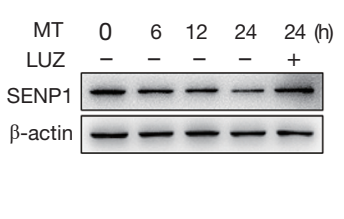

G

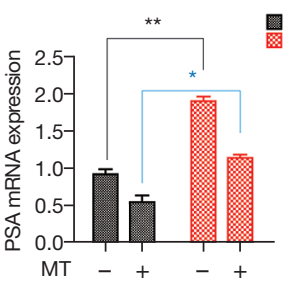

E
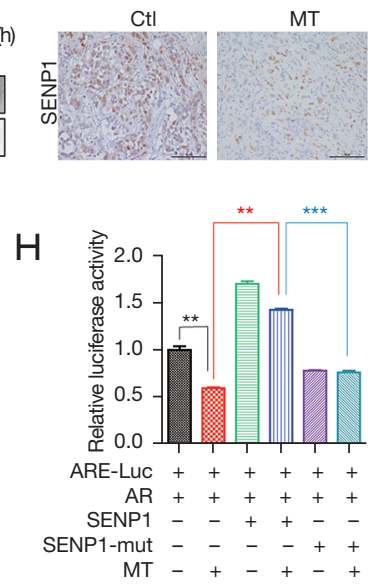

Figure $5 \mathrm{MT}$ decreases the expression of SENP1 in LNCaP cells and xenograft tumor tissue. (A) RT-qPCR analysis of the relative levels of SENP1 in LNCaP cells treated with MT at the indicated doses for $24 \mathrm{~h}$. (B) Western blot analysis showing the protein level of SENP1 in LNCaP cells treated with MT at the indicated doses for 24 h. (C) RT-qPCR analysis and (D) western blot analysis showing the mRNA and protein levels of SENP1 treated with MT $(2 \mathrm{mM})$ or the combination of MT and luzindole $(1 \mu \mathrm{M})$. The time immediately after MT treatment was designed as $0 \mathrm{~h}$ and 6-24 h represent the time points. (E) IHC staining with SENP1 in vehicle- and MT-treated tumors. (F) RT-qPCR analysis and (G) western blot analysis showing the mRNA and protein levels of AR, SENP1, and PSA in LNCaP cells overexpressed SENP1 after treatment with MT (2 mM) for 24 h. (H) Co-transfected with AR expressing vector, ARE-luc plasmid and SENP1 or SENP1mut expressing vector as indicated, 293T cells were exposed to MT (2 mM) as indicated for 48 h. Transfected cells were harvested and assayed for luciferase activity. Data in (A), (C), (F), and (H) are presented as the means \pm SEM. of three independent experiments. ${ }^{*} \mathrm{P}<0.05,{ }^{* *} \mathrm{P}<0.01$, ${ }^{* * *} \mathrm{P}<0.001$ versus control group. MT, melatonin; SENP1, SUMO specific protease 1 ; AR, androgen receptor; ARE, AR-response elements; PSA, prostate-specific antigen; LNCaP, Lymph Node Carcinoma of the Prostate.

prostate cancer cells, the mechanism we illuminated could be expanded to androgen-independent prostate cancer cells.

SUMO was identified as a post-translational protein modifier (50). It is reported that $\mathrm{AR}$ and four of its coregulators (HDAC1, SRC-1, SRC-2, and p300) are conjugated by SUMO $(51,52)$. Generally, SUMOylation has a suppressive effect on AR-dependent transcription; for example, AR SUMOylation of histone deacetylase 4 (HDAC4) decreases the activity of AR (53), and a mutant AR that cannot be SUMOylated has a threefold increase in its transcriptional activity compared to wild-type AR. On the contrary, deSUMOylation of HDAC1 by SENP1 enhances AR transcriptional activity (51). In addition to HDAC1, SUMOylation of AR is also regulated by SENP1 (35), which signifies that the mechanism of MT on AR activity will be not restricted to HDAC1. To maximize efficiency, MT may regulate $\mathrm{AR}$ activity through direct and indirect manners.

SENP1, an important regulator of SUMO modification of target protein, plays an important role in prostate carcinogenesis $(33,38,40,54,55)$. Enhanced SENP1 expression was found not only in early prostate cancer, but was also correlated with the aggressiveness of the disease. By deSUMOylating hypoxia inducible factor $1 \alpha$ (HIF1 $\alpha$, SENP1 could promote prostate cancer invasion and bone metastasis (40). Since MT treatment decreased the expression of SENP1 in LNCaP cells, it was highly suggestive that MT could suppress the proliferation of advanced prostate cancer. Further studies will be necessary to investigate the effect of MT of androgen-independent prostate cancer.

In summary, our data demonstrated that MT functions as a strong inhibitor of AR to markedly reduce AR-dependent transcription. In preliminary studies, we found that SENP1 is decreased in response to MT treatment, resulting in increased SUMOylation of HDAC1, which explains the impaired AR activity. Further studies will be required to determine whether MT can also provide benefits for advanced prostate cancer. 
A

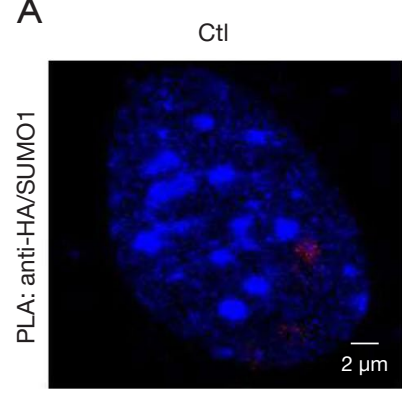

Ctl

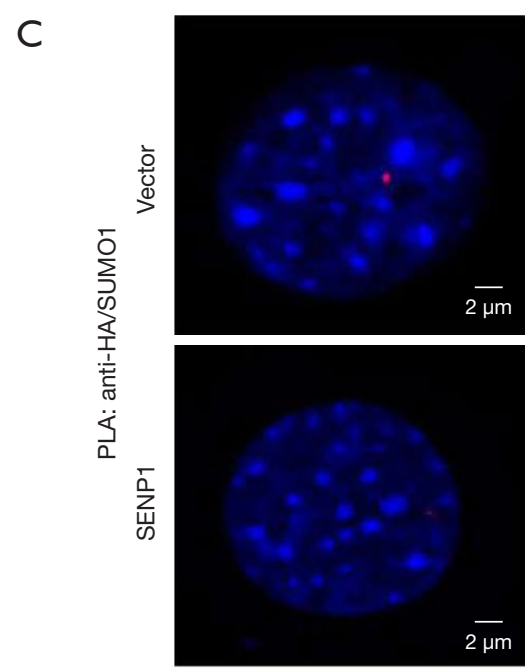

MT

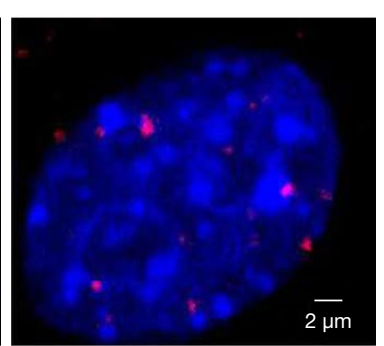

MT

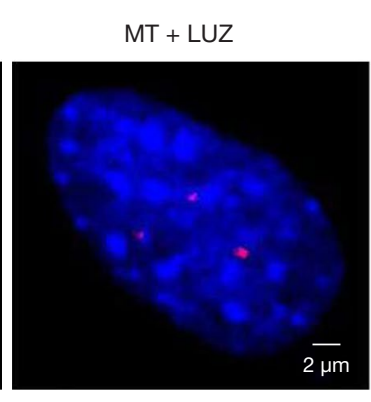

$\mathrm{D}$
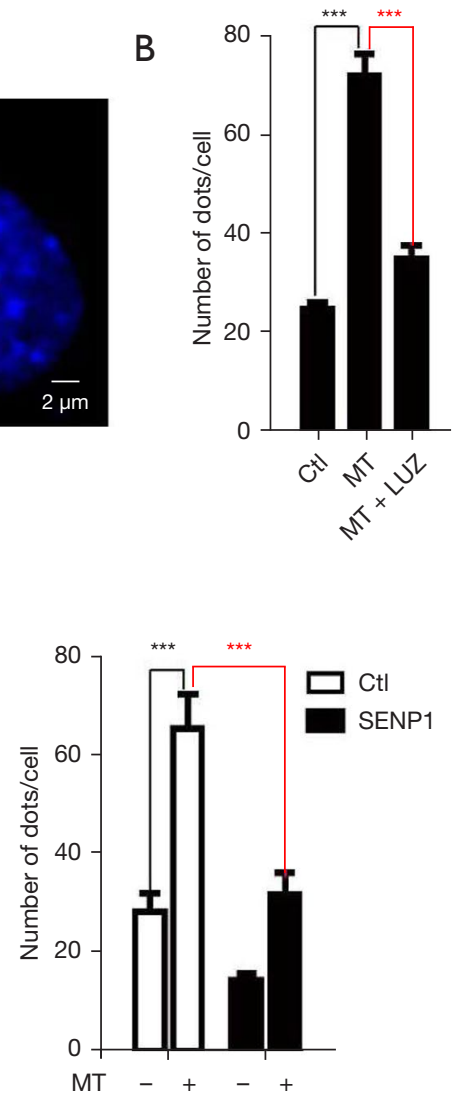

Figure 6 MT increases SUMOylation level of HDAC1. (A) 293T cells were transfected with HA-tagged HDAC1 and Flag-tagged SUMO1 plasmid, cells treated with MT (2 $\mathrm{mM})$ or the combination of MT and luzindole for 24h as indicated. The SUMOylation of HDAC1 was demonstrated using a PLA in 293T cells. DNA was counterstained with (DAPI, blue). Confocal microscopy was used to detect the interaction (red dots). (B) Number of dots corresponding to SUMOylated HDAC1 per cell from each group in (A). (C) Overexpression of SENP1 could counteract the effect of MT on HDAC1 SUMOylation. (D) Number of dots corresponding for SUMOylated HDAC1 per cell from each group in (C). Each bar in (B) and (D) was based on a statistic of 30 imaged cells. ${ }^{* * *} \mathrm{P}<0.001$ versus control group. MT, melatonin; HDAC1, SUMOylation of histone deacetylases 1; PLA, Proximity ligation assay; DAPI, 4',6-diamidino-2-phenylindole.0

\section{Acknowledgments}

Funding: This work was supported by the funds from Jiangsu Province, Key Research and Development program (BE2017635 and BE2015623); Jiangsu Province, Young Medical Talents (QNRC2016386, QNRC2016383, and QNRC2016389); Jiangsu Medical Innovation Team (CXTD-2016-48); Xu Zhou Health Innovation Team (XWCX201603); National Natural Science Foundation of China (81774089); Xuzhou Science and Technology Program (KC18036); Jiangsu Province, Natural Science Research Projects (17KJB360001 and 18KJB180007); and Xuzhou Medical Outstanding Talents [Xuzhou Health
Education Research (2017) No. 22].

\section{Footnote}

Reporting Checklist: The authors have completed the ARRIVE reporting checklist. Available at https://tau. amegroups.com/article/view/10.21037/tau-21-1110/rc

Data Sharing Statement: Available at https://tau.amegroups. com/article/view/10.21037/tau-21-1110/dss

Conflicts of Interest: All authors have completed the ICMJE uniform disclosure form (available at https://tau.amegroups. 
com/article/view/10.21037/tau-21-1110/coif). The authors have no conflicts of interest to declare.

Ethical Statement: The authors are accountable for all aspects of the work in ensuring that questions related to the accuracy or integrity of any part of the work are appropriately investigated and resolved. This study was approved by the Ethics Committee of the Xuzhou Central Hospital (No. XZXY-LJ-20210303-024). The operation process of all experimental animals was strictly in accordance with the "Guide to the Care and Use of Experimental Animals" (2016).

Open Access Statement: This is an Open Access article distributed in accordance with the Creative Commons Attribution-NonCommercial-NoDerivs 4.0 International License (CC BY-NC-ND 4.0), which permits the noncommercial replication and distribution of the article with the strict proviso that no changes or edits are made and the original work is properly cited (including links to both the formal publication through the relevant DOI and the license). See: https://creativecommons.org/licenses/by-nc-nd/4.0/.

\section{References}

1. Hong $Y$, Won J, Lee $Y$, et al. Melatonin treatment induces interplay of apoptosis, autophagy, and senescence in human colorectal cancer cells. J Pineal Res 2014;56:264-74.

2. Mao L, Dauchy RT, Blask DE, et al. Melatonin suppression of aerobic glycolysis (Warburg effect), survival signalling and metastasis in human leiomyosarcoma. J Pineal Res 2016;60:167-77.

3. Ordoñez R, Fernández A, Prieto-Domínguez N, et al. Ceramide metabolism regulates autophagy and apoptotic cell death induced by melatonin in liver cancer cells. J Pineal Res 2015;59:178-89.

4. Farez MF, Mascanfroni ID, Méndez-Huergo SP, et al. Melatonin Contributes to the Seasonality of Multiple Sclerosis Relapses. Cell 2015;162:1338-52.

5. Singh AK, Haldar C. Melatonin modulates glucocorticoid receptor mediated inhibition of antioxidant response and apoptosis in peripheral blood mononuclear cells. Mol Cell Endocrinol 2016;436:59-67.

6. Su LY, Li H, Lv L, et al. Melatonin attenuates MPTPinduced neurotoxicity via preventing CDK5-mediated autophagy and SNCA/ $\alpha$-synuclein aggregation. Autophagy 2015;11:1745-59.

7. Wu HM, Shen QY, Fang L, et al. JNK-TLR9 signal pathway mediates allergic airway inflammation through suppressing melatonin biosynthesis. J Pineal Res 2016;60:415-23.

8. Alonso-González C, González A, Martínez-Campa C, et al. Melatonin sensitizes human breast cancer cells to ionizing radiation by downregulating proteins involved in double-strand DNA break repair. J Pineal Res 2015;58:189-97.

9. Fan C, Pan Y, Yang Y, et al. HDAC1 inhibition by melatonin leads to suppression of lung adenocarcinoma cells via induction of oxidative stress and activation of apoptotic pathways. J Pineal Res 2015;59:321-33.

10. Fan L, Sun G, Ma T, et al. Melatonin overcomes apoptosis resistance in human hepatocellular carcinoma by targeting survivin and XIAP. J Pineal Res 2013;55:174-83.

11. Hill SM, Belancio VP, Dauchy RT, et al. Melatonin: an inhibitor of breast cancer. Endocr Relat Cancer 2015;22:R183-204.

12. León J, Casado J, Jiménez Ruiz SM, et al. Melatonin reduces endothelin-1 expression and secretion in colon cancer cells through the inactivation of FoxO-1 and NF$\kappa \beta$. J Pineal Res 2014;56:415-26.

13. Mayo JC, Hevia D, Quiros-Gonzalez I, et al. IGFBP3 and MAPK/ERK signaling mediates melatonin-induced antitumor activity in prostate cancer. J Pineal Res 2017;62:e12373.

14. Paroni R, Terraneo L, Bonomini F, et al. Antitumour activity of melatonin in a mouse model of human prostate cancer: relationship with hypoxia signalling. J Pineal Res 2014;57:43-52.

15. Su SC, Hsieh MJ, Yang WE, et al. Cancer metastasis: Mechanisms of inhibition by melatonin. J Pineal Res 2017;62:e12370.

16. Wang SW, Tai HC, Tang CH, et al. Melatonin impedes prostate cancer metastasis by suppressing MMP-13 expression. J Cell Physiol. 2021;236:3979-3990.

17. Jan JE, Reiter RJ, Wong PK, et al. Melatonin has membrane receptor-independent hypnotic action on neurons: an hypothesis. J Pineal Res 2011;50:233-40.

18. Yu L, Liang H, Lu Z, et al. Membrane receptor-dependent Notch1/Hes1 activation by melatonin protects against myocardial ischemia-reperfusion injury: in vivo and in vitro studies. J Pineal Res 2015;59:420-33.

19. Maurice P, Daulat AM, Turecek R, et al. Molecular organization and dynamics of the melatonin $\mathrm{MT}_{1}$ receptor/RGS20/G(i) protein complex reveal asymmetry of receptor dimers for RGS and G(i) coupling. EMBO J 2010;29:3646-59. 
20. Mazna P, Grycova L, Balik A, et al. The role of proline residues in the structure and function of human MT2 melatonin receptor. J Pineal Res 2008;45:361-72.

21. Karasek M, Gruszka A, Lawnicka H, et al. Melatonin inhibits growth of diethylstilbestrol-induced prolactinsecreting pituitary tumor in vitro: possible involvement of nuclear RZR/ROR receptors. J Pineal Res 2003;34:294-6.

22. Siegel RL, Miller KD, Jemal A. Cancer statistics, 2016. CA Cancer J Clin 2016;66:7-30.

23. Wang H, Sun D, Ji P, et al. An AR-Skp2 pathway for proliferation of androgen-dependent prostate-cancer cells. J Cell Sci 2008;121:2578-87.

24. Van Asseldonk B, Black P, Elterman DS. Chemical vs Surgical ADT in Metastatic Prostate Cancer: A Comparison of Side Effects. Commentary on Comparison of Gonadotropin-releasing Hormone Agonists and Orchiectomy: Effects of Androgen Deprivation Therapy. Urology 2016;93:3-4.

25. Mitra R, Goodman OB Jr. CYP3A5 regulates prostate cancer cell growth by facilitating nuclear translocation of AR. Prostate 2015;75:527-38.

26. Yu K, Wang RX, Li MH, et al. Melatonin Reduces Androgen Production and Upregulates Heme Oxygenase-1 Expression in Granulosa Cells from PCOS Patients with Hypoestrogenia and Hyperandrogenia. Oxid Med Cell Longev 2019;2019:8218650.

27. Shen D, Ju L, Zhou F, et al. The inhibitory effect of melatonin on human prostate cancer. Cell Commun Signal 2021;19:34.

28. Sigurdardottir LG, Markt SC, Rider JR, et al. Urinary melatonin levels, sleep disruption, and risk of prostate cancer in elderly men. Eur Urol 2015;67:191-4.

29. Bartsch C, Bartsch H, Schmidt A, et al. Melatonin and 6-sulfatoxymelatonin circadian rhythms in serum and urine of primary prostate cancer patients: evidence for reduced pineal activity and relevance of urinary determinations. Clin Chim Acta 1992;209:153-67.

30. Gilad E, Matzkin H, Zisapel N. Inactivation of melatonin receptors by protein kinase $\mathrm{C}$ in human prostate epithelial cells. Endocrinology 1997;138:4255-61.

31. Jung-Hynes B, Schmit TL, Reagan-Shaw SR, et al. Melatonin, a novel Sirt1 inhibitor, imparts antiproliferative effects against prostate cancer in vitro in culture and in vivo in TRAMP model. J Pineal Res 2011;50:140-9.

32. Siu SW, Lau KW, Tam PC, et al. Melatonin and prostate cancer cell proliferation: interplay with castration, epidermal growth factor, and androgen sensitivity. Prostate 2002;52:106-22.
33. Bawa-Khalfe T, Cheng J, Wang Z, et al. Induction of the SUMO-specific protease 1 transcription by the androgen receptor in prostate cancer cells. J Biol Chem 2007;282:37341-9.

34. Kaikkonen S, Jääskeläinen T, Karvonen U, et al. SUMOspecific protease 1 (SENP1) reverses the hormoneaugmented SUMOylation of androgen receptor and modulates gene responses in prostate cancer cells. Mol Endocrinol 2009;23:292-307.

35. Wu R, Cui Y, Yuan X, et al. SUMO-specific protease 1 modulates cadmium-augmented transcriptional activity of androgen receptor (AR) by reversing AR SUMOylation. Toxicol Lett 2014;229:405-13.

36. Cheng J, Kang X, Zhang S, et al. SUMO-specific protease 1 is essential for stabilization of HIF1alpha during hypoxia. Cell 2007;131:584-95.

37. Yu T, Zuo Y, Cai R, et al. SENP1 regulates IFN- $\gamma$-STAT1 signaling through STAT3-SOCS3 negative feedback loop. J Mol Cell Biol 2017;9:144-53.

38. Cheng J, Bawa T, Lee P, et al. Role of desumoylation in the development of prostate cancer. Neoplasia 2006;8:667-76.

39. Yeh ET. SUMOylation and De-SUMOylation: wrestling with life's processes. J Biol Chem 2009;284:8223-7.

40. Wang Q, Xia N, Li T, et al. SUMO-specific protease 1 promotes prostate cancer progression and metastasis. Oncogene 2013;32:2493-8.

41. Luo HR, Liu Y, Wan XD, et al. Sumoylation Negatively Regulates CSR1-Dependent Prostate Cancer Cell Death. Cell Physiol Biochem 2018;46:1861-7.

42. Cos S, Sánchez-Barceló EJ. Melatonin and mammary pathological growth. Front Neuroendocrinol 2000;21:133-70.

43. Lewy AJ, Wehr TA, Goodwin FK, et al. Light suppresses melatonin secretion in humans. Science 1980;210:1267-9.

44. National Research Council (US) Committee for the Update of the Guide for the Care and Use of Laboratory Animals. Guide for the Care and Use of Laboratory Animals. 8th edition. National Academies Press (US); Washington, DC: 2011.

45. Dhanasekaran SM, Barrette TR, Ghosh D, et al. Delineation of prognostic biomarkers in prostate cancer. Nature 2001;412:822-6.

46. Li Z, Alyamani M, Li J, et al. Redirecting abiraterone metabolism to fine-tune prostate cancer anti-androgen therapy. Nature 2016;533:547-51.

47. Shen HC, Balk SP. Development of androgen receptor antagonists with promising activity in castration-resistant 
prostate cancer. Cancer Cell 2009;15:461-3.

48. Qin J, Lee HJ, Wu SP, et al. Androgen deprivationinduced NCoA2 promotes metastatic and castrationresistant prostate cancer. J Clin Invest 2014;124:5013-26.

49. Sharma NL, Massie CE, Ramos-Montoya A, et al. The androgen receptor induces a distinct transcriptional program in castration-resistant prostate cancer in man. Cancer Cell 2013;23:35-47.

50. Dou H, Huang C, Van Nguyen T, et al. SUMOylation and de-SUMOylation in response to DNA damage. FEBS Lett 2011;585:2891-6.

51. Cheng J, Wang D, Wang Z, et al. SENP1 enhances androgen receptor-dependent transcription through desumoylation of histone deacetylase 1. Mol Cell Biol 2004;24:6021-8.

52. Gross M, Yang R, Top I, et al. PIASy-mediated repression

Cite this article as: Hao L, Dong Y, Zhang JJ, He HG, Chen JG, Zhang SQ, Zhang QJ, Wu W, Han CH, Shi ZD. Melatonin decreases androgen-sensitive prostate cancer growth by suppressing SENP1 expression. Transl Androl Urol 2022;11(1):91-103. doi: 10.21037/tau-21-1110 of the androgen receptor is independent of sumoylation. Oncogene 2004;23:3059-66.

53. Yang Y, Tse AK, Li P, et al. Inhibition of androgen receptor activity by histone deacetylase 4 through receptor SUMOylation. Oncogene 2011;30:2207-18.

54. Burdelski C, Menan D, Tsourlakis MC, et al. The prognostic value of SUMO1/Sentrin specific peptidase 1 (SENP1) in prostate cancer is limited to ERG-fusion positive tumors lacking PTEN deletion. BMC Cancer 2015;15:538.

55. Wang C, Tao W, Ni S, et al. Tumor-suppressive microRNA-145 induces growth arrest by targeting SENP1 in human prostate cancer cells. Cancer Sci 2015;106:375-82.

(English Language Editor: A. Kassem) 